\title{
Topic Study Group No. 45: Knowledge in/for Teaching Mathematics at Primary Level
}

\author{
Carolyn A. Maher, Peter Sullivan, Hedwig Gasteiger and Soo Jin Lee
}

As the teaching of primary level mathematics (ages 5-13) is complex, it requires teachers to master a variety of types of knowledge. These are outlined as follows:

Understanding of important mathematical concepts that underpin meaningful student learning of the main strands of the mathematics curriculum;

- Appreciation of the mathematical processes (conceptual understanding, problem solving, and reasoning) in which students engage in doing mathematics, building mathematical arguments, and their justifications of solutions to problems;

- Selecting and building into lessons tasks that engage students in meaningful mathematics and numeracy learning;

- Awareness and knowledge of activities, tasks, and interventions that engage and develop persistence in students while exploring mathematical investigations;

- Awareness and appreciation of the value of tools (manipulatives) and technology in students' building multiple representations of mathematical ideas;

- Awareness of children's development in their learning of mathematical ideas (e.g. place value, number sense, operations) from informal to formal understandings;

Co-chairs: Carolyn A. Maher, Peter Sullivan.

Team members: Hedwig Gasteiger, Soo Jin Lee.

\section{C.A. Maher}

Rutgers University, New Brunswick, USA

e-mail: carolyn.maher@gse.rutgers.edu

P. Sullivan $(\square)$

Monash University, Melbourne, Australia

e-mail: Peter.sullivan@monash.edu

(C) The Author(s) 2017

G. Kaiser (ed.), Proceedings of the 13th International Congress on Mathematical

Education, ICME-13 Monographs, DOI 10.1007/978-3-319-62597-3_72 
- Knowledge of pedagogies that are appropriate with heterogeneous classes including specific actions to support students' learning, such as collaborative group activities;

- Knowledge of resources (collaborative communities, lessons, activities, video collections) to support teacher learning.

TSG 45 participants explored the types of knowledge represented by these various challenges, and how teachers can be supported in their learning.

The major presentations for the group were:

- Professional knowledge for early mathematics education by Hedwig Gasteiger and Christiane Benz

- Supporting teachers in improving their knowledge of mathematics by Peter Sullivan

- Teacher learning about mathematical reasoning: An instructional model by Robert Sigley and Carolyn A. Maher

- What is required for teachers to reorganize math textbooks? - Textbook analysis based on key developmental understandings by Soo Jin Lee and Jaehong Shin

- Using task design to build teacher knowledge by Brenda Bicknell and Jenny Young-Loveridge

- Using tasks from contexts to engage students in meaningful and worthwhile mathematics learning by Doug Clarke and Anna Roche

- Teaching the language of mathematics: What teachers need to know and do by Louise C. Wilkinson

- Structure and development of primary teacher's professional competencies by Dennis Meyer, Andreas Busse, Jessica Hoth, Martina Dohrmann

- Pupils as knowledge agents and monitors in the construction of mathematical ideas by Therese Dooley.

The presentations covered a broad range of topics for supporting the improvement in knowledge for teachers of mathematics. In particular topics addressed included knowledge for:

- teaching mathematics in the early years

- diagnosis and support for students with special needs

- incorporating reasoning in teaching and assessment

- text analysis with a focus on fractions

- the design and use of contextual tasks

- supporting pupil construction of mathematics

- processes and purpose of task design and adaptation

- development and use of appropriate language of mathematics

- structure and algebra in all years

- effective incorporation of measurement into teaching and assessment

- catering for the needs of gifted students

- using the study of patterns as a prompt to abstraction and generalization

- analysis of misconceptions associated with learning decimals 
- the methods of teaching proportional reasoning

- approaches to teaching equivalent fractions

- characteristics of an equitable and balanced curriculum

- providing corrective feedback and analysis of incorrect answers

- listening to and interpreting student thinking

- establishing classrooms as communities of learners and inquiry

- effective representations of mathematics concepts

- processes of teaching students to solve problems

- the connections between culture and classroom processes

- the drama of teaching.

Open Access Except where otherwise noted, this chapter is licensed under a Creative Commons Attribution 4.0 International License. To view a copy of this license, visit http://creativecommons. org/licenses/by/4.0/. 